\section{Does the Hydrated Electron Occupy a Cavity?}

\author{
Ross E. Larsen, ${ }^{*} \dagger$ William J. Glover, $\ddagger$ Benjamin J. Schwartz*
}

Since the discovery of the hydrated electron more than 40 years ago, a general consensus has emerged that the hydrated electron occupies a quasispherical cavity in liquid water. We simulated the electronic structure and dynamics of the hydrated electron using a rigorously derived pseudopotential to treat the electron-water interaction, which incorporates attractive oxygen and repulsive hydrogen features that have not been included in previous pseudopotentials. What emerged was a hydrated electron that did not reside in a cavity but instead occupied a 1-nanometer-diameter region of enhanced water density. Both the calculated ground-state absorption spectrum and the excited-state spectral dynamics after simulated photoexcitation of this noncavity hydrated electron showed excellent agreement with experiment. The relaxation pathway involves a rapid internal conversion followed by slow ground-state cooling, the opposite of the mechanism implicated by simulations in which the hydrated electron occupies a cavity.

$\mathrm{T}$ he nature of excess electrons in liquid water has been of continuing interest due to their important role in radiation chemistry and charge-transfer reactions. Excess electrons can be created directly by pulse radiolysis, or they can be formed after ionization of a solute if the detached electron resides in the liquid far from its parent cation. When liquid water locally contains one more electron than is needed to maintain electrical neutrality, the metastable localized species that is created has been termed the hydrated electron $\left(e_{a q}^{-}\right)$. The hydrated electron has attracted considerable theoretical interest, in part because it poses the intriguing question of how a polar solvent acts to localize an object whose size and shape is determined self-consistently by interaction with its surroundings. Thus, although the hydrated electron is nominally a simple singleelectron species, the many-body nature of its interactions with the surrounding water molecules has made this a nontrivial problem in statistical mechanics and quantum chemistry that can directly confront experiment.

Early continuum and semicontinuum models treated the hydrated electron as a spherical charge distribution with a radius determined selfconsistently by polarization of the surrounding solvent. Such models provided insight into the mechanism by which electrons may be localized but did not give a microscopic picture for the structure of the solvent in the presence of the $e_{a q}^{-}$. Subsequent studies used molecular simulations to address such structural questions; in such simulations, the excess electron was treated quantum-mechanically, the surrounding water molecules were treated classically, and the electronwater interaction was described by what is known

Department of Chemistry and Biochemistry, University of California, Los Angeles, Los Angeles, CA 90095-1569, USA. *To whom correspondence should be addressed. E-mail: Ross.Larsen@nrel.gov (R.E.L.); schwartz@chem.ucla.edu (B.J.S.) †Present address: National Renewable Energy Laboratory, Golden, CO 80401-3305, USA.

$\ddagger$ Present address: Department of Chemistry, Stanford University, Stanford, CA 94305-5080, USA. formally as a pseudopotential (1-3). Although alternatives have been proposed (4), the consensus picture that emerged from such simulations was that a hydrated electron excludes water from a small region, so that the $e_{a q}^{-}$occupies a nearly spherical void that is surrounded by water molecules whose $\mathrm{O}-\mathrm{H}$ bonds point toward the cavity (5). This picture is known as the cavity model of the hydrated electron, and because simulations in which the electron occupies a cavity agree reasonably well with experiment, the cavity model has come to dominate both experimental and theoretical discussions of the $e_{a q}^{-}$(6).

Despite this well-accepted view, important questions still remain about the properties of the hydrated electron. One ongoing controversy is the mechanism by which the $e_{a q}^{-}$relaxes to equilibrium after photoexcitation. The debate is whether relaxation occurs by rapid internal conversion to the ground state followed by slow reequilibration of the surrounding water molecules (7-9) or by slow internal conversion followed by rapid reequilibration on the ground state $(10,11)$. Another concern is that the cavity model predicts a strong anisotropy in polarized transient holeburning experiments $(12,13)$, but this predicted signature is not seen experimentally $(14,15)$.

Here, we describe the results of molecular dynamics simulations based on a new, rigorously derived electron-water pseudopotential. The results of our calculations suggest that the hydrated electron does not occupy a cavity but instead encompasses a region of enhanced water density in which the electronic wavefunction overlaps $\sim 37$ ground- and excited-state properties of this noncavity electron and, in every case, have found that our predictions are consistent with experiment. For certain properties, our simulations offer a better match to experiment than simulations in which the electron resides in a cavity. Thus, we suggest that previous claims that the hydrated electron occupies a cavity may have been premature and that a different physical picture of this important species may be more appropriate. water molecules. We calculated several distinct
A key element in any simulation of the hydrated electron is the electron-water interaction specified by the pseudopotential. Although there is a formalism for deriving pseudopotentials using information from quantum chemistry calculations (16), the challenge lies in representing such pseudopotentials in a functional form that is convenient for molecular simulation. Thus, most bulk hydrated electron simulations have been based on ad hoc electron-water interactions $(6,17)$ or have used pseudopotentials that were constrained to have a simple functional form that neglected important details in the molecularcore region (2). Recently, however, we have shown that it is possible to generate rigorous molecular pseudopotentials directly from the orbitals obtained in quantum chemistry calculations (16). Thus, we decided to reexamine the properties of the hydrated electron using a rigorously derived electron-water pseudopotential (18), a two-dimensional contour plot of which is shown in the left panels of Fig. 1. Some of the notable features of our pseudopotential are the presence of deep attractive wells on each hydrogen atom, a substantial repulsion of the electron between the two hydrogen atoms, and
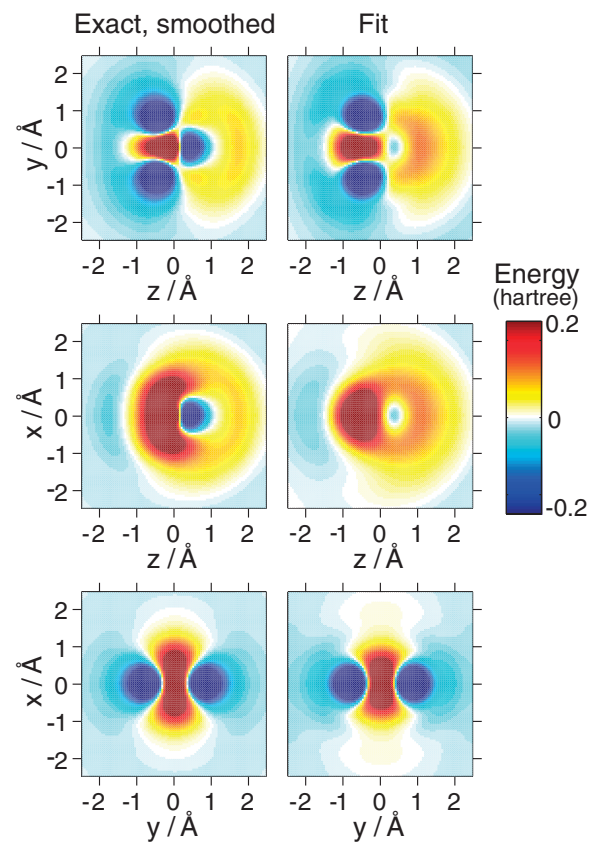

Fig. 1. Cuts of the full smoothed electron-water pseudopotential (left panels) and our fit to the full smoothed pseudopotential (right panels, eq. S5, and table S1); the energy scale is in Hartree. The water molecule lies in the $y-z$ plane with the dipole pointing along the $z$ axis. The 0 atom is at position $y=0.0, z=0.11663 \AA$, and the $H$ atoms are at $y=$ $\pm 0.76001, z=-0.46654 \AA$. The upper panels show a cut parallel to the molecular $y-z$ plane with $x=$ $0.0260 \AA$; the middle panels show a cut perpendicular to the plane of the molecule with $y=0.0260 \AA$ (which is the closest plane to the principal axis of the molecule given our finite grid sampling); the bottom panels show a cut perpendicular to the molecular plane through the two $\mathrm{H}$ atoms. 
an attractive feature near the oxygen atom opposite the hydrogens. Although the presence of the latter two features has been noted in work examining water-cluster anions $(3,19)$, these features have not been incorporated into the pseudopotentials in previous bulk $e_{a q}^{-}$simulations $(1,2)$, so their importance to the behavior of the bulk hydrated electron has not been explored. Thus, to account for the presence of these features, we have fitted our potential to a sum of hydrogen- and oxygencentered terms, plus a sum of terms centered midway between the two hydrogen atoms; the fit is shown in the right panels of Fig. 1, and the fitting parameters and functional form of our pseudopotential are given in table S1.

Using our rigorously determined potential, we have run extensive mixed quantum/classical molecular dynamics (MD) simulations of hundreds of water molecules confined with a single excess electron in a cubic box. The water-water interactions were governed by a flexible simple point charge model (18), and the simulation time step was $0.5 \mathrm{fs}$. The adiabatic eigenstates of the electron were found at every time step on a 32 by 32 by 32 cubic grid that was $18.17 \AA$ on a side using the Lanczos algorithm in the manner described in (20); we verified that the eigenstates did not change by more than $0.03 \mathrm{eV}$ when a $64^{3}$ grid was used. The equilibrium (ground state) results displayed here come from $30 \mathrm{ps}$ of dynamics with 499 water molecules in a box $24.64 \AA$ on a side in the microcanonical ensemble (constant number of molecules, volume, and total energy) at an average temperature of $\sim 300 \mathrm{~K}$. The nonadiabatic, excited-state trajectories were performed with 200 water molecules in a box the same size as the cubic grid. A comparison of the spectroscopic and other properties found with 200 and 499 water molecules is given in figs. S3 and S4.

Figure 2 displays structural and electronic properties of the $e_{a q}^{-}$computed from these simulations. The black dashed and solid curves in Fig. 2A display average values of the hydrated electron's charge density and $r^{2}$ times this density, respectively, relative to the electron's average position, or center-of-mass (COM). These curves show that the hydrated electron is clearly not a point-particle but instead has charge distributed over a $\sim 2.6 \AA$ radius of gyration, in good agreement with the experimental value of $\sim 2.5 \AA$ obtained from spectral moment analysis (21). Fig. 2A also shows radial distribution functions (RDFs), which give the probability of finding either $\mathrm{H}$ atoms (red dashed curve) or $\mathrm{O}$ atoms (solid blue curve) on the surrounding water molecules relative to the electron's COM. Clearly, the electron's charge density has considerable overlap with regions containing a high density of water molecules: A major fraction of the charge density of the $e_{a q}^{-}$is found between the first hydrogen and oxygen peaks in the RDFs. This is seen explicitly in the snapshots shown in Fig. 2, B and C, which show that many water molecules reside inside the volume occupied by the hydrated electron.
The radial distribution functions (RDFs) in Fig. 2A make clear that the hydrated electron does not exclude a discrete volume about itself, so that a description of the $e_{a q}^{-}$as residing in a cavity is inappropriate. Although the RDFs become small at distances close to the electron's $\mathrm{COM}$, the fall-off is gradual, more akin to the solvation structure around a soft sphere rather than a hard excluded volume. Moreover, the RDFs do not reach zero at the origin, indicating that solvent fluctuations occasionally allow water oxygen atoms to penetrate all the way to the hydrated electron's COM. The fact that there is a higher probability for $\mathrm{H}$ atoms to be close to the electron's COM than $\mathrm{O}$ atoms indicates that the electron induces a net orientational ordering of the water molecules inside the electron (an ordering that can also be seen in Fig. 2, B and $\mathrm{C})$, although the preference for water $\mathrm{O}-\mathrm{H}$ bonds to point toward the $\mathrm{COM}$ is reduced from that seen in cavity models of the electron $(2,5,10)$.

The slight maxima and minima in the RDFs show that the electron also induces some translational structural organization of the nearby water molecules, but even the slight minimum in the oxygen $\mathrm{RDF}$ at $\sim 3.5 \AA$ lies above 1 . Integration of the $\mathrm{RDF}$ gives an average number of molecules within $6 \AA$ of the electron $\mathrm{COM}$ of $\sim 37$, whereas
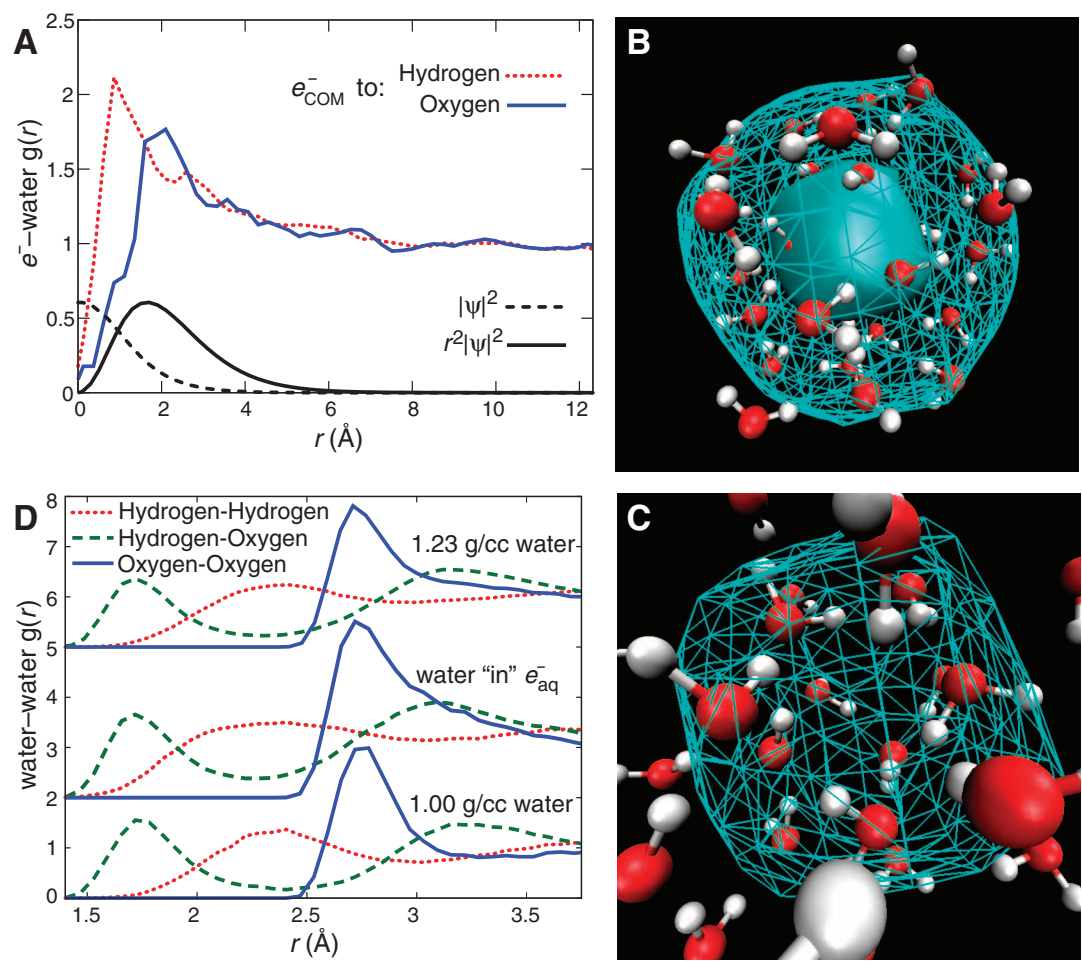

Fig. 2. (A) Electron COM-to-water site radial distribution functions (colored curves). The dashed curve is the square of the wavefunction as a function of distance from the COM, calculated as a time-average over $12.5 \mathrm{ps}$ of dynamics, and the solid curve is this quantity multiplied by $r^{2}$ (both in arbitrary units). (B) All water molecules within $6.0 \AA$ of the COM of the electron for a representative configuration. The wire mesh outer contour encloses $90 \%$ of the electron's charge density, and the opaque inner contour encloses 50\%. (C) Expanded version of the configuration in (B), with the $50 \%$ contour represented by a mesh. (D) Water-water radial distribution functions for pure water at density $1.00 \mathrm{~g} / \mathrm{cm}^{3}$ (lower curves), $1.23 \mathrm{~g} / \mathrm{cm}^{3}$ (upper curves), and for water molecules located within $3.25 \AA$ of the electron center of mass (middle curves). The three sets of curves have been shifted vertically for clarity. 
tron resides in a cavity. First, in our calculations the excited states form a continuous band, unlike cavity models that have three low-lying excited states and then an energy gap below the higherlying, so-called continuum states. Second, in our model, the solvent induces greater fluctuations in the ground-state energy than in the excited-state energies. In calculations with approximate pseudopotentials where the electron resides in a cavity, the fluctuations of the ground- and excited-state energies are of similar magnitude $(1,2,10)$.

We calculated the optical absorption spectrum of the $e_{a q}^{-}$from our simulations by binning the energy gaps weighted by the square of the appropriate transition dipole moment for the lowest seven transitions from configurations taken every $10 \mathrm{fs}$ (18), as shown by the red dotted curve in Fig. 3B. The agreement with the GaussianLorentzian fit to the experimental spectrum (solid black curve) is remarkable: The spectrum calculated with our model matches the experimental spectrum more closely than calculations with pseudopotentials that lead to a cavity-based hydrated electron $(1,2,10)$. Moreover, shifting the experimental spectrum $0.15 \mathrm{eV}$ lower in energy (dashed black curve) shows that the shape of the low-energy edge of the calculated spectrum is correct. We do not expect to reproduce the high-energy tail of the spectrum in these calculations, both because of the small number of excited states we have included (calculations with 20 states on a few configurations show that the tail does begin to fill in) and because we have not accounted for quantum effects in the solvent (22). Fig. 3C shows a decomposition of the calculated absorption spectrum of the $e_{a q}^{-}$into sub-bands associated with transitions from the ground state to each of the lowest seven excited states. As in the cavity model, the bulk of the spectrum comes from transitions to the lowest three excited states. However, because there is no gap between the first three excited states and the higher-lying states, our computed spectrum does not show the unphysical dip between the bright states and the highenergy tail typically seen in spectra predicted by cavity models $(1,2,6,10,17,22)$.

The three lowest excited states that contribute the most to the absorption spectrum in our model of the $e_{a q}^{-}$each have a positive and a negative lobe, as shown for a representative configuration in fig. S1, although the lobes are typically of different sizes. These three lowest-lying quasi$p$-like states are substantially larger than the ground state of the hydrated electron, having average radii of gyration of 4.6(0.3), 5.0(0.5), and 5.4(0.6) $\AA$, in increasing order of energy (root-mean-squared deviations given in parentheses). These radii match well with the estimates from excited-state scavenging experiments (23). Perhaps more interestingly, the three low-lying quasi- $p$-like states do not point in rigorously orthogonal directions: Dot products between the normalized transition dipoles from the ground to the three quasi- $p$-like states, $\cos \theta=\vec{u}_{i} \cdot \vec{u}_{j}$, are distributed roughly as $\exp (-\cos \theta / 0.1)$, as shown in fig. S2. These deviations from strict orthogonality occur because small fluctuations in the positions of water molecules far from the electron COM can alter the orientation of one of the excited states without greatly affecting the other two. This is in sharp contrast to what occurs when the electron occupies a cavity: In that case, the cavity's shape strictly determines the relative orientations of the transition dipoles (supporting online text) $(12,24)$.

In the cavity model, the width of the electron's absorption spectrum reflects fluctuations in the shape of the cavity that change the splitting of three relatively well-separated sub-bands, representing transitions to the three bright $p$-like excited states (24). In contrast, our calculations suggest that the spectrum is broadened by large fluctuations in the ground-state energy, so the underlying individual absorption sub-bands overlap substantially and are not distinct, as shown in Fig. 3C. Each of the three bright sub-spectra spans roughly $3 / 4$ of the width of the entire band, consistent with experimental estimates of the magnitude of homogeneous broadening in the hydrated electron spectrum (25). The fact that the transitions to the different excited states overlap in energy and have transition dipoles that are not strictly orthogonal suggests that there should be no detectable anisotropy in polarized pumpprobe hole-burning measurements on the hydrated electron (15). This is a very different prediction from the strong polarized anisotropy that emerges in simulations based on the cavity model $(12,13)$. Thus, unlike the cavity model, our calculations are consistent with the experimental observations that polarized hole-burning experiments on the $e_{a q}^{-}$show no anisotropy $(14,15)$.

In addition to the ground-state electronic structure, a sound model for the $e_{a q}^{-}$should also be able to correctly predict the relaxation dynamics after photoexcitation to one of the lowlying optically accessible excited states. Thus, we chose to compute the relaxation dynamics of a photoexcited hydrated electron with our model by running 20 nonadiabatic, nonequilibrium trajectories in which the electron is instantaneously resonantly excited by light of energy $1.55 \pm$ $.01 \mathrm{eV}(\sim 800 \mathrm{~nm})(18)$. For probe wavelengths near the excitation wavelength, the red curve in Fig. $4 \mathrm{C}$ shows that the calculated transient dynamics of the $e_{a q}^{-}$involve bleach recovery on both fast (150 fs, $75 \%$ amplitude) and slow (1.1 ps, $25 \%$ amplitude) time scales (black curve). The transient dynamics in this region resemble the time traces of Barbara and co-workers (at a 740-nm pump wavelength, to account for the slight red shift of our calculated spectrum) (7). At wavelengths longer than the excitation pulse, our calculations predict an initial bleach followed by a delayed transient absorption (blue and green curves in Fig. 4C), also similar to the experimental data (11). We note that the magnitude of the delayed absorption should be slightly underestimated by our calculations because we compute transitions only to the lowest seven excited states. Figure 4D shows that this means that the agreement between our calculated transient absorption spectra and experiment is at least as good asand possibly better than - that with the cavity model (dotted blue curve at $t=1 \mathrm{ps}$ ), which requires the calculated spectra to be red-shifted by nearly $0.7 \mathrm{eV}$ to come close to matching the experimental data (dashed blue curve at $t=1 \mathrm{ps}$ ) $(2,12)$.

Simulations in which the electron resides in a cavity suggest that the transient absorption dynamics are governed primarily by solvation of the excited state, with a slow internal conversion followed by fast equilibration of the ground state
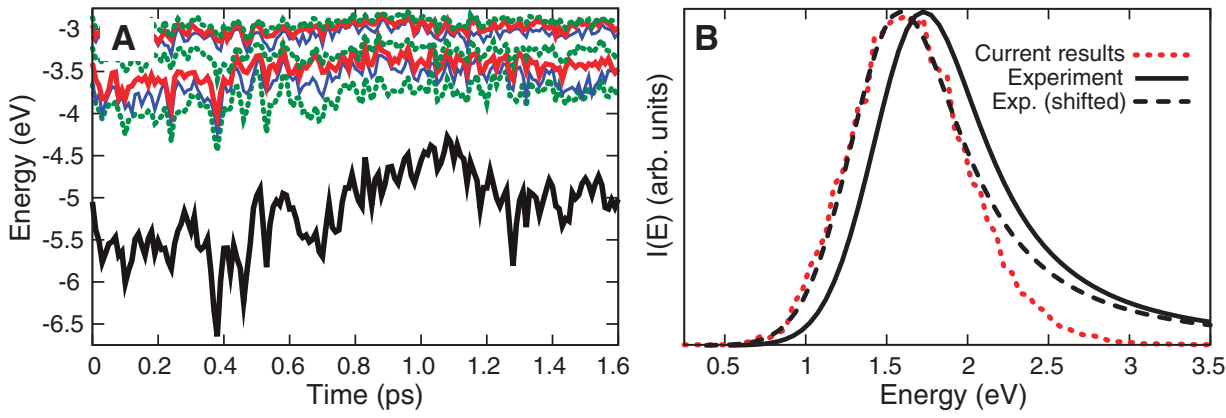

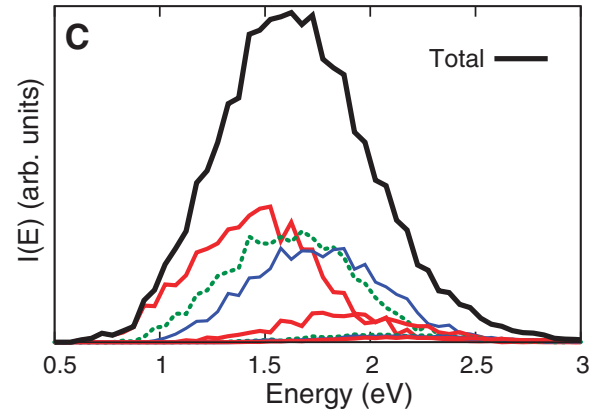

Fig. 3. Equilibrium properties of the hydrated electron. (A) Time evolution of the lowest eight adiabatic energy levels. (B) Calculated absorption spectrum (red dotted curve) from the ground to the lowest seven excited states, analytical fit to the experimental absorption spectrum [black solid curve (30)], and experimental spectrum shifted by $0.15 \mathrm{eV}$ (black dashed curve). (C) Total absorption spectrum (black curve) and absorption spectra associated with excitation to each of the lowest seven adiabatic states (colored curves). 
$(10,12,13)$. This adiabatic picture, however, is not the only way to interpret the observed spectral behavior. Early pump-probe measurements on the $e_{a q}^{-}$were explained in terms of a nonadiabatic model involving rapid internal conversion followed by slow cooling of a nonequilibrium ground state $(7,8)$. Moreover, more recent experiments studying the excitation of water cluster anions also strongly suggest that relaxation occurs by rapid internal conversion followed by slow ground-state cooling (9). The experimental literature on the relaxation of the bulk hydrated electron seems to be about evenly divided between the adiabatic and nonadiabatic relaxation mechanisms.

Figure 4A shows a representative excitedstate trajectory of the hydrated electron simulated with our model. The salient features are that the ground-to-occupied energy gap closes very rapidly [an observation that is consistent
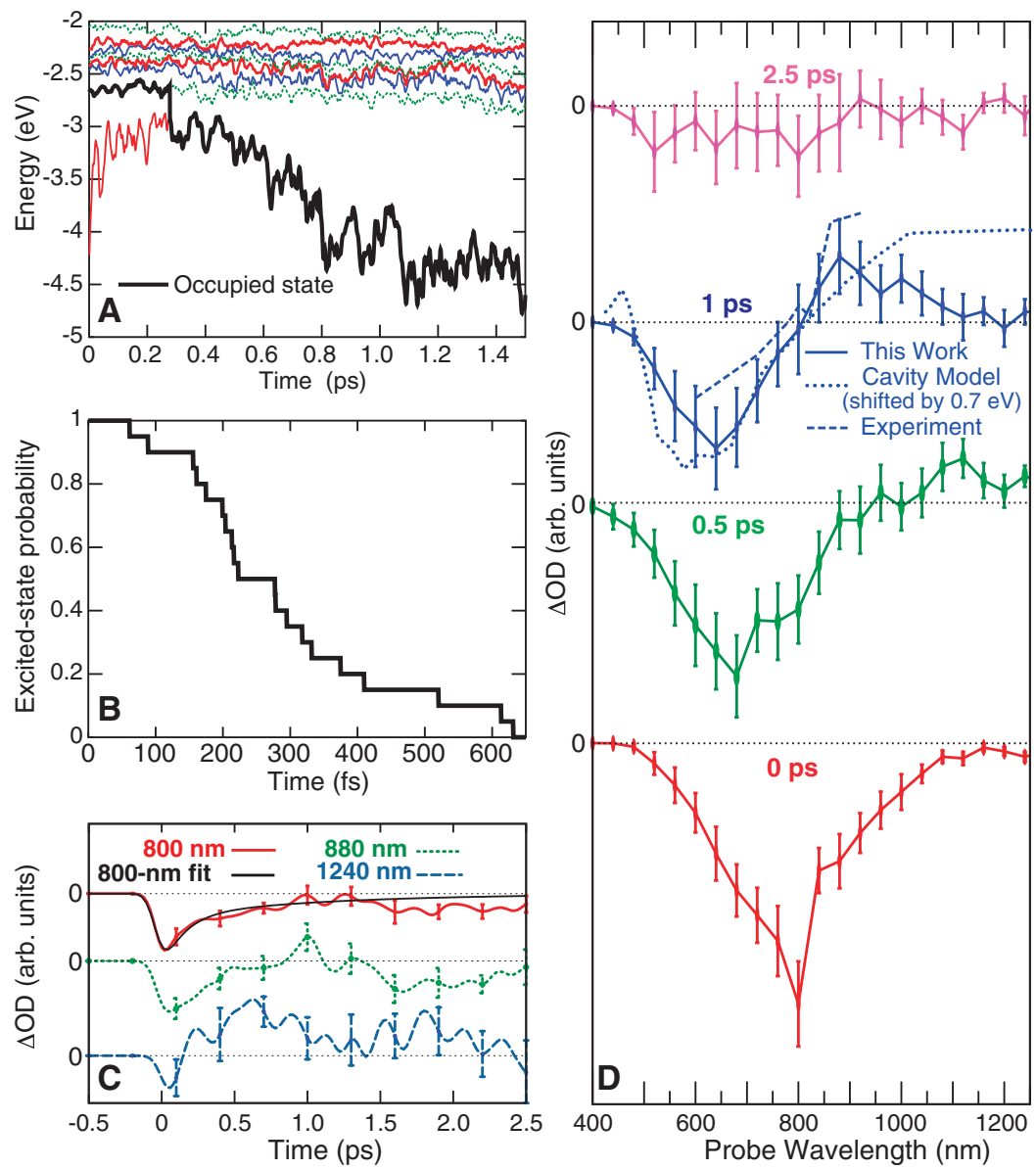

Fig. 4. Nonadiabatic excited-state relaxation dynamics of the hydrated electron. (A) Adiabatic energy levels (colored curves) as a function of time after excitation by $1.55 \mathrm{eV}$, with the heavy black curve indicating the occupied state. (B) The probability for the electron to remain in an excited state as a function of time after excitation for our 20 trajectories. (C) Calculated transient absorption dynamics at 130-fs time resolution for the wavelengths indicated; the curves have been offset vertically for clarity. The black curve shows a fit of our predicted results to a double-exponential decay with time scales of $150 \mathrm{fs}$ and 1100 fs with a 3:1 amplitude ratio, respectively, in excellent agreement with experimental data (7). (D) Simulated transient absorption spectra (solid curves) for the indicated times after excitation, with the curves offset vertically for clarity. Error bars are \pm 1 SD of the mean. The dashed blue curve shows the experimental transient spectrum at 1 ps (11). The dotted blue curve shows the cavity model's simulated transient spectrum at $1 \mathrm{ps}$, shifted by $-0.68 \mathrm{eV}$ to aid comparison to the experiment (13). ulations, the induced absorption results from transitions originating from a nonequilibrated ground state of the $e_{a q}^{-}$, produced after rapid internal conversion. In the cavity model, the delayed absorption comes from so-called $p$-to- $p$ transitions from the occupied first excited state to higher-lying excited states; both the energy gap and the transition dipole magnitudes of these transitions increase over the first few hundred fs (10). Thus, although both models appear to describe the pump-probe data roughly equally well, provided that an empirical shift of the predicted spectra is used for the cavity model, the interpretation of the dynamics underlying the spectral transients in the two models is completely different.

Finally, we note that our results also appear consistent with resonance Raman measurements on the hydrated electron (27), which showed that waters near the $e_{a q}^{-}$have both their bend and stretch frequencies red-shifted relative to those in pure water. Our model suggests that the water molecules that are strongly coupled to the electron are at an effectively higher density than water molecules in the bulk. It is well known that the Raman spectrum of high-density water under pressure shows down-shifts of both the bending and stretching modes, a result of the fact that $\mathrm{O}-\mathrm{H}$ bonds are weakened when protons can be more equally shared between the $\mathrm{O}$ atoms of water molecules that are closer together (28). Thus, part of the major features seen in the Raman spectroscopy of the hydrated electron may result from the change in local water density and structure induced by the presence of the electron. In addition, the strong overlap of the electronic charge density with antibonding molecular orbitals on multiple water molecules also likely plays a role in weakening the $\mathrm{OH}$ bonds (25). The slight orientational ordering of the water molecules inside the electron is also consistent with the Raman spectroscopy, which shows that the two $\mathrm{O}-\mathrm{H}$ bonds of the water molecules coupled to the electron experience different average environments (27).

How should recent ab initio simulations of the $e_{a q}^{-}$be viewed in light of our new model? Boero et al. have performed a DFT-based CarParrinello (CP) study of an excess electron amidst 32 water molecules, which predicted that the hydrated electron occupied a cavity (29), albeit one smaller than those seen in previous cavity-model calculations $(1,2,10)$. The cavity in this CP simulation was transient, however, with a very short lifetime (29). We found that simulations with our model using smaller numbers of water molecules gave larger fluctuations in properties such as the electron's radius of gyration (supporting online text), so it is possible that with only 32 water molecules, the transient cavities Boero et al. observed were due to such fluctuations. Thus, we do not see any inconsistencies between our results and the CP simulations.

\section{References and Notes}

1. J. Schnitker, P. J. Rossky, J. Chem. Phys. 86, 3462 (1987).

2. L. Turi, D. Borgis, J. Chem. Phys. 117, 6186 (2002). 
3. L. D. Jacobson, C. F. Williams, ]. M. Herbert, J. Chem. Phys. 130, 124115 (2009).

4. A. L. Sobolewski, W. Domcke, Phys. Chem. Chem. Phys. 4, 4 (2002).

5. L. Kevan, Acc. Chem. Res. 14, 138 (1981).

6. J. Schnitker, P. J. Rossky, J. Chem. Phys. 131, 037102 (2009).

7. Y. Kimura, ]. C. Alfano, P. K. Walhout, P. F. Barbara, J. Phys. Chem. 98, 3450 (1994).

8. M. S. Pshenichnikov, A. Baltuska, D. A. Wiersma, Chem. Phys. Lett. 389, 171 (2004).

9. A. E. Bragg, ]. R. R. Verlet, A. Kammrath, O. Cheshnovsky, D. M. Neumark, Science 306, 669 (2004).

10. B. J. Schwartz, P. J. Rossky, J. Chem. Phys. 101, 6902 (1994).

11. K. Yokoyama, C. Silva, D. H. Son, P. K. Walhout, P. F. Barbara, J. Phys. Chem. A 102, 6957 (1998)

12. B. J. Schwartz, P. J. Rossky, Phys. Rev. Lett. 72, 3282 (1994).

13. B. J. Schwartz, P. J. Rossky, J. Chem. Phys. 101, 6917 (1994).

14. A. Baltuska, M. F. Emde, M. Pshenichnikov, D. A. Wiersma, J. Phys. Chem. A 103, 10065 (1999).

15. M. C. Cavanagh, I. Martini, B. J. Schwartz, Chem. Phys. Lett. 396, 359 (2004).
16. C. J. Smallwood, R. E. Larsen, W. J. Glover, B. J. Schwartz, J. Chem. Phys. 125, 074102 (2006).

17. R. E. Larsen, W. J. Glover, B. J. Schwartz, J. Chem. Phys. 131, 037101, author reply 037102 (2009).

18. Materials and methods are available as supporting material on Science Online.

19. T. Sommerfeld, A. DeFusco, K. D. Jordan, J. Phys. Chem. A 112, 11021 (2008)

20. R. E. Larsen, M. J. Bedard-Hearn, B. J. Schwartz, J. Phys. Chem. B 110, 20055 (2006).

21. D. M. Bartels, K. Takahashi, J. A. Cline, T. W. Marin, C. D. Jonah, J. Phys. Chem. A 109, 1299 (2005).

22. L. Turi, G. Hantal, P. J. Rossky, D. Borgis, J. Chem. Phys. 131, 024119 (2009).

23. T. W. Kee, D. H. Son, P. Kambhampati, P. F. Barbara, J. Phys. Chem. A 105, 8434 (2001).

24. J. Schnitker, K. Motakabbir, P. J. Rossky, R. A. Friesner, Phys. Rev. Lett. 60, 456 (1988).

25. M. J. Tauber, R. A. Mathies, J. Phys. Chem. A 105, 10952 (2001).

26. S. J. Rosenthal, B. J. Schwartz, P. J. Rossky, Chem. Phys. Lett. 229, 443 (1994).

27. M. J. Tauber, R. A. Mathies, J. Am. Chem. Soc. 125, 1394 (2003).
28. G. E. Walrafen, J. Soln. Chem. 2, 159 (1973)

29. M. Boero, M. Parrinello, K. Terakura, T. Ikeshoji, C. C. Liew, Phys. Rev. Lett. 90, 226403 (2003).

30. F.-Y. Jou, G. R. Freeman, J. Phys. Chem. 83, 2383 (1979).

31. This research was funded by NSF under grant CHE-0908548. We thank M. C. Larsen and A. E. Bragg for helpful discussions, C. N. Mejia for performing the preliminary Hartree-Fock calculations that we used to generate the new electron-water pseudopotential, and K. D. Jordan for a critical reading of the manuscript.

Supporting Online Material

www.sciencemag.org/cgi/content/full/329/5987/65/DC1

Materials and Methods

SOM Text

Figs. S1 to $S 4$

Table S1

References

15 March 2010; accepted 14 May 2010

10.1126/science. 1189588

\section{Experimental Results for $\mathrm{H}_{2}$} Formation from $\mathrm{H}^{-}$and $\mathrm{H}$ and Implications for First Star Formation

H. Kreckel, ${ }^{1 *} \dagger$ H. Bruhns, ${ }^{1} \ddagger$ M. Čížek, ${ }^{2}$ S. C. 0. Glover, $^{3}$ K. A. Miller, ${ }^{1}$ X. Urbain, ${ }^{4}$ D. W. Savin ${ }^{1}$

During the epoch of first star formation, molecular hydrogen $\left(\mathrm{H}_{2}\right)$ generated via associative detachment (AD) of $\mathrm{H}^{-}$and $\mathrm{H}$ is believed to have been the main coolant of primordial gas for temperatures below $10^{4}$ kelvin. The uncertainty in the cross section for this reaction has limited our understanding of protogalaxy formation during this epoch and of the characteristic masses and cooling times for the first stars. We report precise energy-resolved measurements of the AD reaction, made with the use of a specially constructed merged-beams apparatus. Our results agreed well with the most recent theoretically calculated cross section, which we then used in cosmological simulations to demonstrate how the reduced AD uncertainty improves constraints of the predicted masses for Population III stars.

$\mathrm{M}$ ore than 40 years ago, Saslaw and Zipoy (1) proposed that the formation of neutral molecular hydrogen played a central role in the cooling of primordial gas. Collisions of atomic $\mathrm{H}$ with molecular $\mathrm{H}_{2}$ transfer kinetic energy from the $\mathrm{H}$ into internal energy of the $\mathrm{H}_{2}$ through ro-vibrational excitation of the molecule. Subsequently, the molecule can radiatively relax and the emitted photon may escape from the cloud, thereby cooling the gas. Recent numerical models for the formation of the first (Population III) stars have demonstrated the importance of primordial $\mathrm{H}_{2}$ as a coolant (2-4).

${ }^{1}$ Columbia Astrophysics Laboratory, Columbia University, New York, NY 10027, USA. ${ }^{2}$ Charles University Prague, Faculty of Mathematics and Physics, Institute of Theoretical Physics, 18000 Praha 8, Czech Republic. ${ }^{3}$ Zentrum für Astronomie der Universität Heidelberg, Institut für Theoretische Astrophysik, 69120 Heidelberg, Germany. ${ }^{4}$ Institute of Condensed Matter and Nanosciences, Université Catholique de Louvain, Louvain-la-Neuve B-1348, Belgium.

*Present address: Department of Chemistry, University of Illinois, Urbana, IL 61801, USA.

†To whom correspondence should be addressed. E-mail: hkreckel@illinois.edu

‡Present address: INFICON GmbH, D-50968 Cologne, Germany.
During the epoch of first star formation, $\mathrm{H}_{2}$ was formed primarily via the associative detachment $(\mathrm{AD})$ reaction $(3)$,

$$
\mathrm{H}^{-}+\mathrm{H} \rightarrow \mathrm{H}_{2}^{-} \rightarrow \mathrm{H}_{2}+\mathrm{e}^{-}
$$

Although this is the simplest anion-neutral chemical reaction, theory and experiment have failed to reach consensus for both the magnitude and energy dependence of the rate coefficient (5). This uncertainty severely limits our ability to model protogalaxies and metal-free stars forming from initially ionized gas, such as in $\mathrm{H}$ II regions created by earlier Population III stars $(5,6)$. For example, it limits our ability to predict whether a given protogalactic halo can cool and condense on a sufficiently short time scale before it is gravitationally disrupted through a collision with another protogalactic halo (5). Additionally, the cosmological simulations we have performed for this work show that for gas in free fall, the resulting spread in the minimum gas temperatureand in the gas density at which this minimum is reached-leads to a factor of $>20$ difference in the Jeans mass. Finally, for shocked gas under- going isobaric collapse, the time required to reach the cosmic microwave background temperature can differ by up to a factor of 3 . This affects the likelihood that such gas will cool and collapse to form a star before undergoing another shock (6).

The three most recent theoretical calculations for Eq. 1 have not converged, although they all used the same potential for the intermediate $\mathrm{H}_{2}$ anion. The results of Sakimoto (7) and Ćížek et al. (8) are in good agreement with one another but not with the calculations of Launay et al. (9). The room-temperature flowing afterglow results of (10-12), however, are discrepant with the calculations of Sakimoto and Č́žek et al. but are in reasonable agreement with those of Launay et al. See (13) for a brief review. Unfortunately, the existing experimental work provides no constraint on the temperature dependence for Eq. 1, which limits the ability of these measurements to benchmark theory. Considering all these issues, we have designed and built a dedicated mergedbeams apparatus in order to perform energyresolved $\mathrm{AD}$ measurements spanning the entire relevant collision energy range.

An overview of the experiment is shown in Fig. 1; a detailed description is given in (14). In the first leg of the apparatus, we extract an $\mathrm{H}$ beam from a negatively biased ion source to achieve a beam energy $e U_{\mathrm{s}}$ of $\sim 10 \mathrm{keV}$. We collimate it via standard ion beam technology. The atomic $\mathrm{H}$ beam is generated in the central portion of the second leg of the apparatus, where $\sim 7.4 \%$ of the $\mathrm{H}^{-}$is neutralized by photodetachment (PD) with a laser diode array at $975 \mathrm{~nm}$ with continuous-wave power of $1.4 \mathrm{~kW}$. At the photon energy $(1.25 \mathrm{eV})$ and power density used, the PD process produces exclusively ground-state $\mathrm{H}$ atoms. The PD occurs in the center of a drift tube, $1.2 \mathrm{~m}$ in length, used to control the relative energy between the $\mathrm{H}$ and $\mathrm{H}$ beams. When a voltage $-U_{\mathrm{f}}$ is applied to the tube, the $\mathrm{H}^{-}$ions are decelerated as they enter the tube and are then accelerated 